\section{Projeto Bambuí: fatores associados ao conhecimento da condição de hipertenso entre idosos}

\author{
The Bambui Health and Aging Study (BHAS): \\ factors associated with awareness \\ of hypertension among older adults
}

\author{
1 Núcleo de Estudos \\ em Saúde Pública \\ e Envelhecimento, Centro \\ de Pesquisas René Rachou, \\ Fundação Oswaldo Cruzl \\ Universidade Federal \\ de Minas Gerais, \\ Belo Horizonte, Brasil. \\ Correspondência \\ Josélia Oliveira Araújo Firmo \\ Núcleo de Estudos \\ em Saúde Pública \\ e Envelhecimento, Centro \\ de Pesquisas René Rachou, \\ Fundação Oswaldo Cruzl \\ Universidade Federal \\ de Minas Gerais. \\ Av. Augusto de Lima 1715 \\ Belo Horizonte, $M G$ \\ 30190-002, Brasil. \\ firmoj@cpqrr.fiocruz.br
}


tes da busca do tratamento é o conhecimento da condição de hipertenso. Estudos de base populacional realizados em países desenvolvidos e em desenvolvimento mostram que cerca de $1 / 4$ a 1/3 dos hipertensos não sabiam dessa sua condição 10,11,12,13,14,15. A definição dos fatores associados ao conhecimento da condição de hipertenso é muito importante para orientar estratégias adequadas ao controle da hipertensão arterial. Entretanto, estudos que investigam os fatores associados ao conhecimento da condição de hipertenso na população idosa são raros 11,16 .

O Projeto Bambuí é um estudo de coorte de base populacional da população idosa residente na cidade de Bambuí, Minas Gerais 6. Na linha de base desta coorte, a prevalência da hipertensão arterial era alta (62\%) e somente $63 \%$ dos idosos hipertensos estavam sendo tratados 17. Essa população apresenta prevalência da hipertensão semelhante ao observado em outras comunidades $(61,5 \%)$, estando $62,9 \%$ dos hipertensos em tratamento e destes, $27,0 \%$ apresentando pressão sistólica $<140 \mathrm{mmHg}$ e pressão diastólica $<90 \mathrm{mmHg}$. O presente trabalho é parte da linha de base da coorte de Bambuí e foi desenvolvido com o objetivo de determinar os fatores (sócio-demográficos, hábitos de vida, indicadores da condição de saúde, uso de serviços de saúde e uso de medicamentos) associados ao conhecimento da condição de hipertenso entre idosos residentes na comunidade.

\section{Metodologia}

\section{Área estudada}

O Município de Bambuí possuía 20.573 habitantes em 1991, 15.032 (73\%) dos quais vivendo na sua sede (cidade de Bambuí) ${ }^{3}$. As principais causas de morte neste município em 1996 eram as doenças cerebrovasculares (CID-10: I60-I69), doença de Chagas (CID-10: B57), doenças isquêmicas do coração (CID-10: I20-I25) e doença pulmonar obstrutiva crônica (CID-10: J43J44), com taxas de mortalidade iguais a 110,0 , $61,4,42,5$ e 18,9 por 100 mil habitantes, respectivamente 18. Bambuí foi uma importante área endêmica para doença de Chagas. Apesar da transmissão da infecção pelo Trypanosoma cru$z i$ ter sido interrompida há cerca de vinte anos, a prevalência da infecção permanece alta na população idosa devido a efeito de coorte 19 .

\section{População estudada}

Os participantes da coorte foram identificados por meio de um censo completo da cidade de Bambuí, conduzido pela nossa equipe entre novembro e dezembro de 1996 . Todos os residentes com sessenta ou mais anos de idade foram selecionados para o estudo (1.742 habitantes). Destes, $1.606(92,2 \%)$ foram entrevistados e $1.494(85,8 \%)$ foram submetidos a exames físicos e laboratoriais. Os participantes da linha de base eram semelhantes à população da cidade nesta faixa etária em relação a todas as características sócio-demográficas consideradas, tais como: sexo, idade, renda, escolaridade, etc. 7 . Para o presente trabalho foram selecionados todos os participantes da linha de base da coorte de Bambuí identificados como hipertensos $(n=909)$.

\section{Medidas de pressão arterial}

As medidas de pressão arterial foram realizadas no Posto Avançado de Estudos Emmanuel Dias, da Fundação Oswaldo Cruz (FIOCRUZ), em Bambuí, por técnicos treinados. O treinamento dos técnicos incluiu a utilização de vídeos, ausculta dupla e exercícios para aferição de variabilidade inter e intra-observadores.

Foram realizadas três medidas de pressão arterial, após cinco minutos de descanso, a intervalos de dois minutos, com o indivíduo sentado e com o braço em repouso, na altura do coração. Essas medidas foram realizadas em um recinto isolado, silencioso e com temperatura ambiente controlada. A primeira medida foi feita após pelo menos trinta minutos sem ingestão de café e/ ou uso de cigarro. A pressão arterial foi considerada como a média da segunda e terceira medidas 20,21. Os seguintes instrumentos foram utilizados: esfigmomanômetros Tycos' 509730 (Estados Unidos) e estetoscópios Littman's Cardiology II (Estados Unidos).

Foram considerados hipertensos aqueles que: (1) apresentaram pressão sistólica $\geq 140$ $\mathrm{mmHg}$ e/ou pressão diastólica $\geq 90 \mathrm{mmHg} 20$ e/ou (2) relataram o uso de medicamentos para hipertensão nos últimos noventa dias. As seguintes classes terapêuticas foram consideradas: beta bloqueador, diurético, bloqueador dos canais de cálcio, inibidor da enzima conversora da angiotensina, antagonista da enzima conversora da angiotensina, alfa bloqueador central e/ou vasodilatador 17,22. 


\section{Variável dependente}

A variável dependente deste estudo foi o conhecimento da condição de hipertenso. Considerou-se que sabia ser hipertenso aquele que respondeu afirmativamente à pergunta: "algum médico ou profissional de saúde já disse que o(a) senhor(a) tinha pressão alta ou hipertensão?".

\section{Variáveis independentes}

As variáveis independentes deste estudo foram: (1) características sócio-demográficas (idade, sexo, escolaridade, estado civil e viver só); (2) hábitos de vida (consumo atual de cigarros, freqüência de consumo atual de bebidas alcoólicas, consumo diário de frutas e vegetais frescos nos últimos 12 meses, prática de atividades físicas nas horas de lazer nos últimos noventa dias e tentativa de perder peso nos últimos $12 \mathrm{me}$ ses); (3) indicadores de condição de saúde (relato de diagnóstico médico de diabetes, de angina pectoris, de infarto do miocárdio, história familiar de doenças cardiovasculares antes dos cinqüenta anos de idade, ter estado acamado nas últimas duas semanas, ter deixado de realizar atividade habitual por problemas de saúde nas últimas duas semanas e percepção da própria saúde nos últimos seis meses); (4) uso de serviços de saúde (tempo decorrido após a última medida de pressão arterial, número de consultas médicas nos últimos 12 meses, número de internações hospitalares nos últimos 12 meses); (5) uso de medicamentos (número de medicamentos, prescritos e não prescritos, utilizados nos últimos noventa dias, excluindo-se os medicamentos para hipertensão) e (6) outros (necessidade de respondente próximo na entrevista, presença de cuidador em caso de doença e filiação a plano privado de saúde). Além desses, foram também considerados: índice de massa corporal (peso/altura 2 ), colesterol total após jejum de 12 horas e sorologia para o T. cruzi.

As informações correspondentes aos itens 1 a 6 foram obtidas por meio de entrevistas. As entrevistas foram realizadas no domicílio do participante utilizando-se perguntas estruturadas e pré-codificadas. Os entrevistadores eram moradores da cidade de Bambuí, com pelo menos 11 anos de escolaridade e foram treinados pela equipe do projeto. Quando o entrevistado estava impossibilitado de responder à entrevista devido a déficit cognitivo ou a algum problema de saúde, um respondente próximo adequado foi utilizado 7 .

As medidas antropométricas e a coleta de sangue após jejum de 12 horas foram realiza- das no Posto Avançado de Estudos Emmanuel Dias, em Bambuí. As medidas antropométricas foram realizadas por técnicos especialmente treinados, com os indivíduos usando roupas leves, utilizando-se balança e antropômetro padronizados (CMS Portable Stadiometer Kit, Reino Unido). Para o presente trabalho foram considerados o peso e a altura para a determinação do índice de massa corporal (peso/altu$\mathrm{ra}^{2}$ ). A determinação do nível de colesterol foi feita após jejum de 12 horas, utlizando-se o aparelho Eclipse Vitalab (Merck, Holanda). A presença de anticorpos para o T. cruzi foi determinada por meio das reações de hemaglutinação indireta (Biolab) e ELISA (Abbott, Brasil), considerando-se positivos aqueles que apresentaram anticorpos em ambos os exames. Maiores detalhes podem ser vistos em publicação anterior 7 .

\section{Análise dos dados}

As características dos indivíduos que sabiam ser hipertensos foram comparadas às daqueles que desconheciam esta condição. A análise bivariada dos dados foi baseada no teste do quiquadrado de Pearson ou no teste do qui-quadrado para tendências lineares 23 . Na análise multivariada, as odds ratios e respectivos intervalos de confiança obtidos pelo método de Wolff 24 foram calculados utilizando-se o método de regressão logística múltipla 25 . O critério para inclusão de variáveis no modelo logístico inicial foi a associação com o conhecimento da condição de hipertenso na análise univariada em nível inferior a 0,20 26. Foram mantidas no modelo logístico final as variáveis que apresentaram associações com o conhecimento da condição de hipertenso em nível inferior a 0,05. As análises foram feitas utilizando-se o programa Stata.

\section{Resultados}

Participaram deste estudo 1.494 idosos, que correspondem a $85,8 \%$ de todos os residentes na cidade de Bambuí com sessenta ou mais anos de idade. Destes, 919 (61,5\%) foram classificados como hipertensos, sendo que 215 $(23,4 \%)$ não sabiam ser hipertensos.

Entre os hipertensos, 248 (27,0\%) apresentavam pressão sistólica $<140 \mathrm{mmHg}$ e pressão diastólica < 90mmHg, $570(62,0 \%)$ apresentavam pressão sistólica entre 140 e $159 \mathrm{mmHg}$ e/ou pressão diastólica entre 90 e $99 \mathrm{mmHg}$ e $101(11,0 \%)$ apresentavam pressão sistólica $\geq$ $160 \mathrm{mmHg}$ e/ou pressão diastólica $\geq 100 \mathrm{mmHg}$. 
Cerca de $1 / 3$ dos hipertensos $(37,1 \%)$, não estavam sendo tratados para hipertensão arterial.

A distribuição do conhecimento da condição de hipertenso entre idosos, de acordo com características sócio-demográficas está apresentada na Tabela 1 . Somente sexo e faixa etária apresentaram associações significantes com saber ser hipertenso.

A existência de um cuidador informal quando necessário não apresentou associação com conhecer $(94,7 \%)$ ou não $(93,5 \%)$ a condição de hipertenso ( $\mathrm{p}=0,475)$.

Com relação a hábitos de vida, o saber ser hipertenso esteve significativamente associado à freqüência de consumo de bebida alcoólica nos últimos 12 meses e à tentativa de perder peso neste período (Tabela 2 ).

A associação entre a distribuição do conhecimento da condição de hipertenso e alguns indicadores de condição de saúde pode ser observada na Tabela 3. Percepção da própria saúde, ter estado acamado nas últimas duas semanas, ter deixado de realizar alguma atividade habitual por problemas de saúde nas duas últimas semanas, história médica de diabetes, de Angina pectoris e/ou infarto do miocárdio, história familiar de doenças cardiovasculares antes dos cinqüenta anos de idade, índice de massa corporal e soropositividade para o T. cruzi apresentaram associações significantes com saber ser hipertenso.

A Tabela 4 apresenta a distribuição do conhecimento da condição de hipertenso entre idosos, segundo o uso de serviços de saúde, de medicamentos e filiação a plano privado de saúde. Número de consultas médicas nos últimos 12 meses, número de medicamentos (exceto anti-hipertensivos) usados nos últimos três meses, tempo decorrido após a última medida de pressão arterial e filiação a plano privado de saúde apresentaram associações significantes com saber ser hipertenso.

Após ajustamento por variáveis de confusão, as seguintes características permaneceram significantes e positivamente associadas ao conhecimento da condição de hipertenso: sexo feminino ( $\mathrm{OR}=2,04$; IC95\% $=1,45-2,87$ ), tentativa de perder peso nos últimos 12 meses $(\mathrm{OR}=$ 1,86; IC95\% = 1,14-3,04), percepção da saúde como ruim (OR = 1,93; IC95\% = 1,16-3,20), menor tempo (< 1 ano) decorrido após a última medida da pressão arterial $(\mathrm{OR}=2,97$; IC95\% = 1,76-5,03) e número de consultas médicas (1-3 e $\geq 4$ ) nos últimos 12 meses (OR = 2,14; IC95\% = 1,34-3,41 e OR = 2,23; IC95\% = 1,28-3,86, respectivamente). Associação significante e negativa foi encontrada para faixa etária $\geq 80$ anos $(\mathrm{OR}=0,40 ;$ IC95\% $=0,24-0,68)$ (Tabela 5).

\section{Discussão}

Os resultados deste trabalho mostram uma alta prevalência do desconhecimento da condição de hipertenso entre idosos residentes na comunidade. A prevalência encontrada $(23,4 \%)$ foi semelhante às observadas em estudos utilizando a mesma metodologia na Holanda (20,0\%) 27 e na Espanha $(26,0 \%) 28$, e inferior ao observado em estudos conduzidos na Índia $(55,0 \%)$ 16, Israel (50,0\%) 29, Estados Unidos (37,0\%) $11 \mathrm{e}$ Itália $(34,0 \%) 30$.

Pelo nosso conhecimento, são raros os estudos que investigaram os fatores associados ao conhecimento da hipertensão arterial entre idosos residentes na comunidade. Um desses trabalhos foi desenvolvido em cinco estados do Sudoeste dos Estados Unidos, em uma amostra representativa de 3 mil mexicanos com 65 ou mais anos de idade 11. As seguintes características apresentaram associações independentes

Tabela 1

Distribuição do conhecimento da condição de hipertenso entre idosos, segundo características sociodemográficas selecionadas. Bambuí, Minas Gerais, Brasil, 1997.

\begin{tabular}{|c|c|c|}
\hline \multirow[t]{2}{*}{ Variáveis } & \multicolumn{2}{|c|}{ Sabia ser hipertenso (\%) } \\
\hline & $\operatorname{Sim}(n=704)$ & Não $(n=215)$ \\
\hline \multicolumn{3}{|l|}{ Sexo } \\
\hline Masculino & 29,3 & 53,0 \\
\hline \multirow[t]{2}{*}{ Feminino } & 70,7 & 47,0 \\
\hline & \multicolumn{2}{|c|}{$p<0,001$} \\
\hline \multicolumn{3}{|l|}{ Faixa etária (em anos) } \\
\hline $60-69$ & 59,5 & 55,8 \\
\hline 70-79 & 32,2 & 30,2 \\
\hline \multirow[t]{2}{*}{$\geq 80$} & 8,3 & 14,0 \\
\hline & \multicolumn{2}{|c|}{$\mathrm{p}^{\star}<0,001$} \\
\hline \multicolumn{3}{|l|}{ Estado civil } \\
\hline Casado/mora junto & 47,0 & 49,8 \\
\hline Solteiro/divorciado/separado & 14,8 & 20,0 \\
\hline \multirow[t]{2}{*}{ Viúvo } & 38,2 & 30,2 \\
\hline & \multicolumn{2}{|c|}{$p=0,051$} \\
\hline \multicolumn{3}{|l|}{ Escolaridade (em anos) } \\
\hline Nunca estudou & 32,8 & 33,5 \\
\hline $1-3$ & 33,9 & 26,5 \\
\hline $4-7$ & 26,6 & 31,6 \\
\hline \multirow[t]{2}{*}{$\geq 8$} & 6,7 & 8,4 \\
\hline & \multicolumn{2}{|c|}{$p^{\star}=0,180$} \\
\hline \multicolumn{3}{|l|}{ Morar só } \\
\hline Não & 18,2 & 17,3 \\
\hline \multirow[t]{2}{*}{ Sim } & 81,8 & 82,7 \\
\hline & \multicolumn{2}{|c|}{$p=0,753$} \\
\hline
\end{tabular}

$\mathrm{p}=$ teste do Qui-quadrado de Pearson;

$\mathrm{p}^{\star}=$ teste do Qui-quadrado para tendências lineares. 
Distribuição do conhecimento da condição de hipertenso entre idosos, segundo hábitos de vida selecionados. Bambuí, Minas Gerais, Brasil, 1997.

\begin{tabular}{|c|c|c|}
\hline \multirow[t]{2}{*}{ Variáveis } & \multicolumn{2}{|c|}{ Sabia ser hipertenso (\%) } \\
\hline & $\operatorname{Sim}(n=704)$ & Não $(n=215)$ \\
\hline \multicolumn{3}{|l|}{ Fuma atualmente } \\
\hline Não & 85,2 & 80,0 \\
\hline \multirow[t]{2}{*}{ Sim } & 14,8 & 20,0 \\
\hline & \multicolumn{2}{|c|}{$p=0,067$} \\
\hline \multicolumn{3}{|c|}{ Consumo $\geq 5$ doses diárias de bebida alcoólica nos últimos 12 meses } \\
\hline Não bebeu nos últimos 12 meses & 93,3 & 84,2 \\
\hline$<1 \mathrm{vez} / \mathrm{semestre}-<1 \mathrm{vez} / \mathrm{mês}$ & 3,0 & 7,0 \\
\hline 3 vezes/mês - 2 vezes/semana & 2,8 & 5,6 \\
\hline \multirow[t]{2}{*}{$\geq 3$ vezes/semana } & 0,9 & 3,2 \\
\hline & \multicolumn{2}{|c|}{$p<0,001$} \\
\hline \multicolumn{3}{|c|}{$\begin{array}{l}\text { Prática de atividades físicas durante períodos de lazer }{ }^{1} \\
\text { nos últimos } 90 \text { dias }\end{array}$} \\
\hline Não & 86,6 & 85,1 \\
\hline \multirow[t]{2}{*}{ Sim } & 13,4 & 14,9 \\
\hline & \multicolumn{2}{|c|}{$p=0,586$} \\
\hline \multicolumn{3}{|c|}{ Consumo diário de frutas e vegetais frescos } \\
\hline Não & 49,2 & 55,4 \\
\hline \multirow[t]{2}{*}{ Sim } & 50,8 & 44,6 \\
\hline & \multicolumn{2}{|c|}{$p=0,111$} \\
\hline \multicolumn{3}{|c|}{ Tentativa de perder peso nos últimos 12 meses } \\
\hline Não & 76,8 & 88,8 \\
\hline Sim & 23,2 & 11,2 \\
\hline
\end{tabular}

$\mathrm{p}=$ teste do Qui-quadrado de Pearson:

1 por pelo menos 3 vezes por semana, 20-30 minutos.

com o conhecimento da condição de hipertenso: história positiva de doenças cardiovasculares, percepção da própria saúde como ruim, ausência de fonte regular de cuidados médicos, não estar casado, sexo feminino e ter visitado mais o médico no último ano (duas ou mais vezes). O outro trabalho foi realizado em uma amostra representativa de 1.023 idosos (sessenta ou mais anos de idade), residentes em cinco cidades de Bangladesh e Índia. Neste trabalho verificou-se que uma ou mais visitas ao médico no último ano, maior escolaridade (10 ou mais anos) e sexo feminino apresentavam associações independentes com o conhecimento da condição de hipertenso 16.

De uma maneira geral, os resultados do presente trabalho são consistentes com o observado nos estudos acima mencionados, ou seja, o conhecimento da condição de hipertenso foi mais freqüente entre as mulheres, entre aqueles que usaram mais serviços de saúde (mais consultas medicas e menor tempo decorrido após a última medida de pressão arterial) e en- tre os que percebiam sua saúde como pior. Além desses, encontrou-se associação positiva entre saber ser hipertenso e tentativa de perder peso, e associação negativa com a maior faixa etária.

As mulheres, em comparação aos homens, apresentam maior probabilidade de estar em tratamento e de ter a sua pressão arterial melhor controlada 17,29,31,32, além de saber ser hipertensa, como previamente mencionado 11,16. É de se esperar que o uso de serviços de saúde também aumente a chance de conhecimento da condição de hipertenso, uma vez que o seu uso aumenta a probabilidade de realização do diagnóstico. Neste aspecto, é interessante salientar que na comunidade estudada, o uso de serviços de saúde aumenta a chance de tratamento da hipertensão arterial 17. Com relação à pior percepção da própria saúde, não é possível saber neste e em estudo seccional anterior 11, se essa percepção antecede ou é conseqüência do diagnóstico da hipertensão arterial. Somente estudos de coorte podem esclarecer 
Distribuição do conhecimento da condição de hipertenso entre idosos, segundo alguns indicadores de condição de saúde. Bambuí, Minas Gerais, Brasil, 1997.

\begin{tabular}{|c|c|c|}
\hline \multirow[t]{2}{*}{ Variáveis } & \multicolumn{2}{|c|}{ Sabia ser hipertenso (\%) } \\
\hline & $\operatorname{Sim}(n=704)$ & Não $(n=215)$ \\
\hline \multicolumn{3}{|c|}{ Percepção da própria saúde nos últimos 6 meses } \\
\hline Muito boa/boa & 18,5 & 35,8 \\
\hline Razoável & 49,3 & 45,6 \\
\hline \multirow[t]{2}{*}{ Ruim/muito ruim } & 32,2 & 18,6 \\
\hline & \multicolumn{2}{|c|}{$p^{*}<0,001$} \\
\hline \multicolumn{3}{|c|}{ Ter estado acamado nas 2 últimas semanas } \\
\hline Não & 89,2 & 94,4 \\
\hline \multirow[t]{2}{*}{ Sim } & 10,8 & 5,6 \\
\hline & \multicolumn{2}{|c|}{$p=0,022$} \\
\hline \multicolumn{3}{|c|}{$\begin{array}{l}\text { Ter deixado de realizar alguma atividade habitual por problemas } \\
\text { de saúde nas } 2 \text { últimas semanas }\end{array}$} \\
\hline Não & 80,6 & 89,3 \\
\hline \multirow[t]{2}{*}{ Sim } & 19,4 & 10,7 \\
\hline & \multicolumn{2}{|c|}{$p=0,003$} \\
\hline \multicolumn{3}{|c|}{ Diagnóstico médico anterior de diabetes } \\
\hline Não & 85,0 & 94,4 \\
\hline \multirow[t]{2}{*}{ Sim } & 15,0 & 5,6 \\
\hline & \multicolumn{2}{|c|}{$p<0,001$} \\
\hline \multicolumn{3}{|c|}{ Diagnóstico médico anterior de angina e/ou infarto } \\
\hline Não & 84,7 & 91,2 \\
\hline \multirow[t]{2}{*}{ Sim } & 15,3 & 8,8 \\
\hline & \multicolumn{2}{|c|}{$p=0,016$} \\
\hline \multicolumn{3}{|c|}{$\begin{array}{l}\text { História familiar de doenças cardiovasculares antes } \\
\text { dos } 50 \text { anos de idade }\end{array}$} \\
\hline Não & 49,1 & 58,5 \\
\hline \multirow[t]{2}{*}{ Sim } & 50,9 & 41,5 \\
\hline & \multicolumn{2}{|c|}{$p=0,019$} \\
\hline \multicolumn{3}{|c|}{ Índice de massa corporal $\left(\mathrm{Kg} / \mathrm{cm}^{2}\right)$} \\
\hline$\leq 24$ & 36,6 & 50,2 \\
\hline $25-29$ & 40,5 & 34,0 \\
\hline \multirow[t]{2}{*}{$\geq 30$} & 22,9 & 15,8 \\
\hline & \multicolumn{2}{|c|}{$\mathrm{p}^{\star}<0,001$} \\
\hline \multicolumn{3}{|c|}{ Colesterol total (mg/d) } \\
\hline$<200$ & 22,0 & 24,2 \\
\hline $200-239$ & 33,5 & 33,9 \\
\hline \multirow[t]{2}{*}{$\geq 400$} & 44,5 & 41,9 \\
\hline & \multicolumn{2}{|c|}{$p=0,437$} \\
\hline \multicolumn{3}{|c|}{ Seropositividade para o $T$. cruzi } \\
\hline Negativo & 60,6 & 70,9 \\
\hline Positivo & 39,4 & 29,1 \\
\hline & & \\
\hline
\end{tabular}

$p=$ teste do Qui-quadrado de Pearson; $p^{\star}=$ teste do Qui-quadrado para tendências lineares. 
Distribuição do conhecimento da condição de hipertenso entre idosos, segundo o uso de serviços de saúde, uso de medicamentos e uso de plano privado de saúde. Bambuí, Minas Gerais, Brasil, 1997.

\begin{tabular}{|c|c|c|}
\hline \multirow[t]{2}{*}{ Variáveis } & \multicolumn{2}{|c|}{ Sabia ser hipertenso (\%) } \\
\hline & $\operatorname{Sim}(n=704)$ & Não $(n=215)$ \\
\hline \multicolumn{3}{|c|}{ Número de consultas médicas nos últimos 12 meses } \\
\hline 0 & 10,8 & 34,9 \\
\hline $1-3$ & 52,0 & 42,8 \\
\hline \multirow[t]{2}{*}{$\geq 4$} & 37,2 & 22,3 \\
\hline & \multicolumn{2}{|c|}{$\mathrm{p}^{\star}<0,001$} \\
\hline \multicolumn{3}{|c|}{ Número de internações hospitalares nos últimos 12 meses } \\
\hline 0 & 76,4 & 76,7 \\
\hline 1 & 15,8 & 18,6 \\
\hline \multirow[t]{2}{*}{$\geq 2$} & 7,8 & 4,7 \\
\hline & \multicolumn{2}{|c|}{$p=0,208$} \\
\hline \multicolumn{3}{|c|}{$\begin{array}{l}\text { Número de medicamentos usados nos últimos } 3 \text { meses, prescritos } \\
\text { e não prescritos (exceto anti-hipertensivos) }\end{array}$} \\
\hline 0 & 15,9 & 32,6 \\
\hline $1-2$ & 37,8 & 36,7 \\
\hline $3-4$ & 29,4 & 21,4 \\
\hline \multirow[t]{2}{*}{$\geq 5$} & 16,9 & 9,3 \\
\hline & \multicolumn{2}{|c|}{$p^{\star}<0,001$} \\
\hline \multicolumn{3}{|c|}{ Tempo decorrido após a última medida de pressão arterial (em anos) } \\
\hline$\geq 1$ & 5,5 & 26,1 \\
\hline \multirow[t]{2}{*}{$<1$} & 94,5 & 73,9 \\
\hline & \multicolumn{2}{|c|}{$p<0,001$} \\
\hline \multicolumn{3}{|c|}{ Filiação a plano privado de saúde } \\
\hline Não & 78,3 & 81,4 \\
\hline \multirow[t]{2}{*}{ Sim } & 21,7 & 18,6 \\
\hline & \multicolumn{2}{|c|}{$p=0,324$} \\
\hline
\end{tabular}

$p=$ teste do Qui-quadrado de Pearson; $\mathrm{p}^{\star}=$ teste do Qui-quadrado para tendências lineares.

este aspecto. A associação positiva encontrada no presente trabalho entre tentativa de perder peso e conhecimento da condição de hipertenso, pode estar refletindo a adesão a métodos complementares de tratamento da hipertensão, como dieta e exercícios físicos.

Por outro lado, a explicação para a menor freqüência de conhecimento da condição de hipertenso entre idosos mais velhos não é evidente. Nas investigações anteriormente mencionadas, conduzidas entre idosos americanos, mexicanos e indianos 11,16, não foram encontradas diferenças no conhecimento da condição de hipertenso entre as diferentes faixas etárias. Em outro estudo, conduzido na linha de base da coorte de Bambuí, não foi encontrada associação entre idade e tratamento da hipertensão arterial 17. Duas hipóteses poderiam ser levantadas para explicar a menor freqüência de conhecimento da condição de hipertenso entre idosos mais velhos: (a) menor uso de servi- ços de saúde e (b) imprecisão da informação devido a respondente próximo. Em diferentes países verifica-se que as consultas a médicos no consultório, hospital ou através de telefone diminuem com a idade, ao passo que as consultas no domicílio aumentam 33. Isto é devido à diminuição da mobilidade física e capacidade de locomoção entre idosos mais velhos. A necessidade de respondentes próximos durante as entrevistas aumenta com a idade (no presente trabalho, esta proporção aumentou de $2,2 \%$ aos $60-69$ anos para $6,2 \%$ aos $70-79$ e $9,1 \%$ aos 80 e mais anos de idade) e a qualidade da resposta pode ser diferencial quando da utilização ou não de um respondente próximo na entrevista. Entretanto, é importante ressaltar que a associação negativa entre conhecimento da condição de hipertenso e idade, persistiu mesmo após ajustamentos por indicadores de uso de serviços de saúde e necessidade de respondente próximo durante a entrevista, além 
Resultados estatisticamente significantes ${ }^{1}$ da análise multivariada dos fatores associados ao conhecimento da condição de hipertenso entre idosos. Bambuí, Minas Gerais, Brasil, 1997.

\begin{tabular}{lc}
\hline Variáveis & OR $_{\text {ajustada }}$ (IC95\%) \\
\hline Sexo & 1,00 \\
$\quad$ Masculino & $2,04(1,45-2,87)$ \\
$\quad$ Feminino & \\
Faixa etária (em anos) & 1,00 \\
$\quad 60-69$ & $0,98(0,67-1,43)$ \\
$70-79$ & $0,40(0,24-0,68)$ \\
$\geq 80$ & \\
Percepção da própria saúde & 1,00 \\
$\quad$ Muito boa/boa & $1,44(0,96-2,15)$ \\
$\quad$ Razoável & $1,93(1,16-3,20)$ \\
Ruim/muito ruim & \\
Tentativa de perder peso nos últimos 12 meses & 1,00 \\
$\quad$ Não & $1,86(1,14-3,04)$ \\
Sim & \\
Número de consultas médicas nos últimos 12 meses & \\
0 & $2,97(1,69-4,93)$ \\
$1-3$ & $2,14(1,34-3,41)$ \\
$\geq 4$ & \\
Tempo decorrido após a última medida de pressão arterial (em anos) & \\
$\geq 1$ & $1,76-5,03)$ \\
$<1$ & \\
\hline
\end{tabular}

$1 \mathrm{p}<0,005$.

$\mathrm{p}=$ valor de $\mathrm{p}$ (teste do Qui-quadrado de Pearson); $\mathrm{OR}_{\text {ajustada }}\left(\mathrm{IC}_{\mathrm{C}} \mathrm{5 \%}\right)$ ) odds ratio (intervalo de confiança em nível de 95\%) ajustada por regressão logística múltipla por todas as variáveis listadas na tabela (919 indivíduos participaram da análise final).

das demais variáveis listadas na Tabela 5. Dessa forma, não é razoável supor que nossos resultados pudessem ser explicados por esses fatores. Investigações mais profundas são necessárias para a compreensão deste resultado.

Apesar da alta prevalência da hipertensão arterial entre os idosos na comunidade estudada, ainda é alta a porcentagem daqueles que não sabiam ser hipertensos. O uso de serviços de saúde (número de consultas médicas no úl- timo ano e tempo decorrido após a última medida de pressão arterial) foi o fator modificável mais fortemente associado a este desconhecimento. Este resultado chama a atenção para a importância: (1) do acesso do idoso aos serviços de saúde para que a sua condição de hipertenso possa ser diagnosticada e tratada e (2) da informação prestada por este serviço ao idoso para que este, sabendo ser hipertenso, possa aderir satisfatoriamente ao tratamento. 


\section{Resumo}

O objetivo deste estudo foi determinar os fatores associados ao conhecimento da condição de hipertenso entre idosos residentes na comunidade. Foram selecionados todos os 919 idosos ( $\geq 60$ anos) hipertensos participantes da linha de base da coorte de Bambuí, Minas Gerais, Brasil. Destes, somente 76,6\% sabiam ser hipertensos. As seguintes variáveis apresentaram associações positivas e independentes com saber ser hipertenso:sexo feminino $(O R=2,04 ; 145-2,87)$, percepção da saúde como ruim/muito ruim $(O R=1,93 ; 1,16-3,20)$, tentativa de perder peso nos últimos 12 meses $(O R=$ 1,86;1,14-3,04), número de consultas médicas neste periodo $(1-3:$ OR $=2,14,1,34-3,41 ; \geq 4: O R=2,23,1,76$ $5,03)$ e menor tempo decorrido após a última medida da pressão arterial (OR = 2,97, 1,69-4,93). Associação negativa foi encontrada para faixa etária $\geq 80$ anos $(O R=0,40,0,24-0,68)$. Estes resultados chamam a atenção para a importância: (1) do acesso dos idosos aos serviços de saúde para que a sua condição de hipertenso possa ser diagnosticada e tratada e (2) da informação prestada por este serviço ao idoso para que este, sabendo ser hipertenso, possa aderir satisfatoriamente ao tratamento.

Idosos; Hipertensão;Conhecimento

\section{Referências}

1. United Nations. World population projections to 2125. Popul Dev Rev 1998; 24:183-9.

2. Kumar V. Ageing in India-an overview. Indian J Med Res 1997; 106:257-64.

3. Fundação Instituto Brasileiro de Geografia e Estatística. Anuário Estatístico do Brasil. Censo Demográfico de 1992. Rio de Janeiro: Fundação Instituto Brasileiro de Geografia e Estatística; 1992.

4. Fundação Instituto Brasileiro de Geografia e Estatística. Projeção da população do Brasil por sexo e idade para o período de 1980-2050 - Revisão 2000. Rio de Janeiro: Fundação Instituto Brasileiro de Geografia e Estatística; 2000.

5. Caldwell JC. Population health in transition. Bull World Health Organ 2001; 79:159-60.

6. Lima-Costa MF, Guerra HL, Barreto SM, Guimarães RM. Diagnóstico da situação de saúde da população idosa brasileira: um estudo da mortalidade e das internações hospitalares públicas. Inf Epidemiol SUS 2000; 9:23-41.

7. Lima-Costa MFF, Uchôa E, Guerra HL, Firmo JOA, Vidigal PG, Barreto SM. The Bambuí Health and Ageing Study (BHAS). Methodological approach and preliminary results of a population-based cohort study of the elderly in Brazil. Rev Saúde Pública 2000; 34:126-35.

8. Rudd P. Clinicians and patients with hypertension: unsettled issues about compliance. J Am Heart Assoc 1995; 130:572-9.

9. National Hight Blood Pressure Education Program Working Group. National High Blood Pressure Education Program Working Group in the Elderly. Hypertension 1994; 23:275-85

\section{Colaboradores}

J. O. A. Firmo foi coordenadora do trabalho de campo responsável pela análise dos dados e redação do trabalho. M. F. Lima-Costa foi a orientadora do trabalho, tendo sido responsável pelo delineamento do estudo, supervisão da análise dos dados e da redação do trabalho. E. Uchôa teve participação fundamental na discussão do desenho do estudo, dos resultados e revisão crítica final do artigo.
10. Fuentes R, Iimaniemi N, Laurikainen E, Tuomilehto J, Nissinen A. Hypertension in development economies: a review of population-based studies carried out from 1980-1998. J Hypertension 2000; 18:521-9.

11. Satish S, Markides KS, Zhang D, Goodwin JS. Factors influencing unawareness of hypertension among older Mexican Americans. Prev Med 1997; 26:645-50.

12. Kannel WB. Cardioprotection and antihypertensive therapy: the key importance of addressing the associated coronary risk factors (The Framingham Experience). Am J Cardiol 1996; 77:6B$11 \mathrm{~B}$.

13. Burt VL, Whelton P, Roccella EJ, Brown C, Cutler JA, Higgins M, et al. Prevalence of hypertension in the US adult population. Results from the Third National and Nutrition Examination Survey, 1988-1991. Hypertension 1995; 25:305-13.

14. Whelton PK. Epidemiology of hypertension. Lancet 1994; 344:101-6.

15. Ford ES, Harel Y, Heath G, Cooper RS, Caspersen CJ. Test characteristics of self-report hypertension among the Hispanic population: findings from Hispanic Health and Nutritional Examination Survey. J Clin Epidemiol 1990; 43:159-65.

16. Hypertension Study Group. Prevalence, awareness, treatment and control of hypertension among the elderly in Bangladesh and India: a multicentre study. Bull World Health Organ 2001; 79:490-500

17. Firmo JOA, Barreto SM, Lima-Costa MF. The Bambuí Health and Aging Study (BHAS): factors 
associated with the treatment of hypertension in older adults in the community. Cad Saúde Pública $2003 ; 19: 817-27$.

18. Lima-Costa MF, Barreto S, Uchôa E, Firmo JOA, Vidigal PG, Guerra HL. The Bambuí Health and Aging Study (BHAS): prevalence of risk factors and use of preventive health care services. Rev Panam Salud Pública 2001; 9:219-27.

19. Lima-Costa MF, Barreto SM, Guerra HL, Firmo JOA, Uchôa E, Vidigal PG. Ageing with Trypanosoma cruzi infection in a community where the transmission has been interrupted: the Bambuí Health and Ageing Study (BHAS). Int J Epidemiol 2001; 30:887-93.

20. The fifth report of the Joint National Committee on Detection, Evaluation, and Treatment of High BP. Arch Intern Med 1993; 153:154-83.

21. Kaplan NM. Systemic hypertension: mechanisms and diagnosis. In: Braunwald E, editor. Heart disease: a textbook of cardiovascular medicine. 4 th Ed. Philadelphia: W. B. Saunders Co.; 1992. p. 817-51.

22. Barreto SM, Passos VMA, Firmo JOA, Guerra HL, Vidigal PG, Lima-Costa MF. Hypertension and clustering of cardiovascular risk factors in a community in Southeast Brazil - The Bambuí Health and Ageing Study. Arq Bras Cardiol 2001; 6:576-81.

23. Armitage P, Berry G. Statistical methods in medical research. Oxford: Blackwell Scientific Publications; 1987.

24. Schlesselman JJ. Case control studies. Design, conduct analysis. New York: Oxford University Press; 1982.

25. Hosmer DW, Lemenshow S. Applied logistic regression. New York: Johns Wiley and Sons; 1989.
26. Greenland S. Modeling and variable selection in epidemiologic analysis. Am J Public Health 1989; 79:340-9.

27. van Rossun CTM, van de Mheen $H$, Witteman JCM, Hofman A, Mackenbach JP, Grobbee DE. Prevalence, treatment, and control of hypertension by sociodemographic factors among the Dutch elderly. Hypertension 2000; 35:814-21.

28. Compañ L, Vioque J, Hernández-Aguado I, Quiles J. Factores asociados al conocimiento, tratamiento y control de la hipertensión arterial en poplacion adulta de la Comunidad Valenciana. Aten Primaria 1998; 21:527-33.

29. Burstyn M, Shpilberg O, Ginsberg GM, Cohen A, Stessnan J. Hypertension in the Jerusalem 70 year olds study population: prevalence, awareness, treatment and control. Isr J Med Sci 1996; 32:62933.

30. Prencipe M, Casini AR, Santini M, Ferreti C, Scaldaferri N, Culasso F. Prevalence, awareness, treatment and control of hypertension in the elderly: results from a population survey. J Hum Hypertension 2000; 14:825-30.

31. Satish S, Stroup-Benham CA, Espino DV, Markides KS, Goodwin JS. Undertreatment of hypertension in older Mexican Americans. J Am Geriatr Soc 1998; 46:405-10.

32. Plasencia A, Ostfeld AM, Gruber SB. Effects of sex on differences in awareness, treatment, and control of high blood pressure. Am J Prev Med 1988; 4:315-26.

33. Rowland D. A five-nation perspective on the elderly. Health Affairs 1992; 11:205-16.

Recebido em 24/Abr/2003

Versão final reapresentada em 29/Ago/2003

Aprovado em 23/Out/2003 